Décadrages Décadrages

cınéma, a travers champs Cinéma, à travers champs

$25 \mid 2013$

Werner Herzog

\title{
Aux limites du cadre : Werner Herzog n'est pas Alfred Hitchcock
}

\section{Brad Prager}

Traducteur : Charlotte Bouchez

\section{OpenEdition \\ Journals}

Édition électronique

URL : http://journals.openedition.org/decadrages/719

DOI : 10.4000/decadrages.719

ISSN : 2297-5977

Éditeur

Association Décadrages

Édition imprimée

Date de publication : 15 octobre 2013

Pagination : 8-20

ISBN : 978-2-9700668-7-3

ISSN : 2235-7823

\section{Référence électronique}

Brad Prager, «Aux limites du cadre : Werner Herzog n'est pas Alfred Hitchcock», Décadrages [En ligne], 25 | 2013, mis en ligne le 10 décembre 2015, consulté le 04 mai 2019. URL : http://

journals.openedition.org/decadrages/719; DOI : 10.4000/decadrages.719

(B) Décadrages 
1 En allemand, l'échange de propos était formulé ainsi: "Haben Sie als schauspielernder Regisseur die Vorbilder Alfred Hitchcock und Otto Preminger?" W. H.: "Daran habe ich noch nie gedacht, nein. In eigenen Filmen tauche ich übrigens nicht als Schauspieler auf, das versuche ich zu vermeiden". (Moritz von Uslar, "99 Fragen an Werner Herzog", Die Zeit (ZEITmagazin), no 16, 13 avril 2003, www.zeit. de/2013/16/99-fragen-werner-herzog/).

$2 \mathrm{Ndtr}$ : le terme anglais cameo désigne, dans le contexte cinématographique ou télévisuel, l'apparition d'une personnalité notable sous forme de clin d'œil; il peut être traduit en français par "brève apparition". La traduction littérale serait "camée" qui ne possède pas ce sens particulier, et qui renvoie à une pratique de taille d'une pierre caractérisée par le fait qu'elle présente plusieurs strates de couleurs (ce qui constitue un "camaïeu"). Traditionnellement, le motif du camée est associé au portrait de personnes illustres. La relation sémantique entre le camée comme œuvre de joaillerie et son utilisation dans le contexte cinématographique repose sur le fait qu'ils forment des représentations de personnages célèbres. Nous avons donc choisi d'utiliser le terme cameo tel quel dans le texte en français.
A la question suivante, posée par le journal allemand Die Zeit à Werner Herzog: «En tant qu'acteur-réalisateur, Alfred Hitchcock et Otto Preminger sont-ils vos modèles ?", Herzog répondit: "Je n'ai jamais réfléchi à cela; non. Dans mes propres films, je n'apparais pas comme acteur. C'est quelque chose que j'essaie d'éviter»1. Cette affirmation est, dans une certaine mesure, sujette à caution. Nous savons en effet que Herzog apparaît dans plusieurs de ses films. A ce propos, il est possible de mettre cette pratique en relation avec celle qui caractérise le style propre à Hitchcock; l'un comme l'autre ont tendance à empreindre leurs films de leur présence physique. Alors que la voix et le corps de Herzog sont omniprésents dans nombre de ses documentaires, il apparaît également dans certains de ses films de fiction. Nous pouvons ainsi interroger les ressemblances de ces cas d'auto-mise en scène avec la pratique qui prévaut chez Hitchcock. Ses apparitions, vêtu d'un uniforme de soldat dans Lebenszeichen (Signes de vie, R.F.A., 1968), ou sous les traits d'un ouvrier transportant du verre rubis dans Herz aus Glas (Cour de verre, R.F.A., 1976), sont hitchcockiennes à leur façon; mais que signifie cette similarité? Ces brèves apparitions $\left(\operatorname{cameos}^{2}\right)$ ressemblent-elles simplement à celles de Hitchcock, ou font-elles consciemment écho au style du maître, y compris dans une perspective critique? L'analyse de ces cas nous permettra de préciser la conception que Herzog a de lui-même en tant qu'auteur. Dans une perspective comparative, il s'agira de montrer comment ces deux réalisateurs ont choisi de poser les limites du cadre, manifestant ainsi la façon dont ils délimitent le monde réel qu'ils habitent et les mondes créés dans leurs films.

Lors de certaines apparitions au sein de ses propres films, Herzog n'interprète pas ses rôles comme un véritable acteur; il ne s'engage pas dans une performance, du moins pas au sens conventionnel du terme. De plus, il ne constitue pas un élément essentiel de la diégèse: ces apparitions peuvent ainsi être considérées comme des cameos, au sens pre- 
mier du terme. En effet, le terme cameo, provenant originellement du mot «camée», est généralement associé aux notions de reconnaissance et d'identification. Mais, si le rôle joué par Herzog est secondaire - ses apparitions n'ayant pas d'importance significative dans la diégèse -, alors que fait-il dans ses films (la question se pose également pour Hitchcock, ou n'importe quel autre réalisateur qui interpréterait un rôle ornemental similaire)? Dans le cas de Hitchcock, ses apparitions sont parfois interprétées comme une puissance de déstabilisation ${ }^{3}$. A suivre Christian Metz, ses cameos peuvent être considérés comme donnant à voir l'artificialité de la frontière entre l'histoire - la fiction telle qu'elle se déploie et dans laquelle nous sommes censés être immergés - et le discours, où le film constitue un message que le réalisateur transmet au public ${ }^{4}$. En d'autres termes, les apparitions du réalisateur, parce qu'elles désignent une réalité extérieure au monde du film, nuisent potentiellement à la participation du spectateur ou à l'illusion collective engendrée par le film. Partant, ce type d'apparition du réalisateur constitue un jeu qui entre en contradiction avec l'immersion du spectateur dans la fiction. Si tel est le cas, cette perturbation peut toutefois provoquer un certain plaisir, puisqu'elle indique que le quatrième mur peut être brisé : il pourrait s'effondrer, mais le réalisateur choisit de ne pas le faire, renonçant à la possibilité d'un aparté théâtral explicite. Dans ce sens, le cameo manifeste l'autorité du réalisateur, ou, comme l'écrit Thomas Leitch, il rappelle au public «le pouvoir du metteur en scène» et le fait que le film constitue «un artefact ou un discours artistique plutôt qu'un récit transparent»5. Les spectateurs qui sont conscients de l'apparition du réalisateur peuvent ainsi ressentir un plaisir lié au fait que le réalisateur montre qu'il peut interférer, tout en choisissant de laisser cette option à l'état latent, de ne pas conférer à son interruption un sens explicite.

Le cameo peut ainsi être considéré comme une menace, source de plaisir potentiel, qui se porte à l'encontre de la distinction entre l'histoire et le discours : il se fonde sur le plaisir ressenti par le spectateur à la perspective d'une déstabilisation potentielle ou imaginaire du récit. Cette perspective d'analyse ne souligne qu'un aspect de l'équation qui concerne le fonctionnement du cameo en regard du développement linéaire du récit. Pourtant, ces apparitions forment également des images délibérément prévues, situées, et stylisées dans l'ensemble du film. Il est ainsi possible d'étudier non seulement le fait que le réalisateur apparaisse à l'écran, mais également d'observer là où son corps apparaît, s'il est situé au centre de l'image ou à sa périphérie. Hitchcock peut, le plus souvent, être aperçu en train de se déplacer d'un bord à l'autre de l'écran, son corps prenant ainsi la mesure de toute la largeur du
3 Thomas Leitch, "Games Hitchcock Plays", dans David Boyd (éd.), Perspectives on Alfred Hitchcock, New York, G.K. Hall \& Co, 1995, p. 54.

4 Voir Thomas Leitch, op. cit., p. 55. Leitch reprend les termes histoire et discours employés par Christian Metz dans Le Signifiant imaginaire: psychanalyse et cinéma, Paris, Union générale d'éditions, 1977.

5 Leitch, ibid. 
6 Voir, par exemple, les commentaires de Hitchcock dans Eric Sherman, Directing the Film: Film Directors on their Art, Los Angeles, Acrobat Books, 1976, p. 99.

7 Murray Pomerance, "Some Hitchckockian Shots", dans Thomas Leitch and Leland Poague (éd.), A Companion to Alfred Hitchcock, Malden, Wiley-Blackwell, 2011, p. 238.

8 Paul Cronin (éd.), Herzog on Herzog, Londres, Faber and Faber, 2002, p. 104 [notre traduction]. champ. Par exemple, dans I Confess (La loi du silence, E.-U., 1953) il se place au sommet d'une volée d'escaliers, traversant de gauche à droite et dans sa totalité l'espace du cadre. Dans Vertigo (Sueurs froides, E.-U., 1958), il traverse également l'écran, mais cette fois en sens inverse. Dans les séquences de ce type, Hitchcock marche d'un bord à l'autre du cadre, comme s'il démontrait sa connaissance précise des coordonnées du plateau. Ainsi, son apparition constitue davantage un geste de stabilisation que de déstabilisation, puisqu'elle manifeste la conscience qu'a le réalisateur des limites du cadre et de la maîtrise de ses frontières. A la manière d'un peintre chinois qui se représente dans le paysage parfaitement maîtrisé qu'il a réalisé, les apparitions d'Hitchcock sont parfaitement et proportionnellement ordonnées : son cadrage est si méticuleusement construit que le réalisateur peut y apparaître, montrant au spectateur comment il est possible d'en prendre précisément la mesure.

Appréhender le cinéma comme on approche le cadre d'un tableau conduit à envisager la précision du story-board comme la manifestation d'un contrôle. Hitchcock est connu pour avoir toujours élaboré scrupuleusement ses productions en amont de la réalisation, œuvrant plus comme un architecte que comme un impressionniste, chaque angle de vue étant soumis à une coordination algébrique. Bien qu'il prétende ne pas toujours se reposer sur des story-boards $\mathbf{6}$, quiconque a vu ces documents pourra témoigner de sa remarquable précision. Comme le fait remarquer Murray Pomerance à propos des cadrages de Hitchcock, chaque aspect de leur composition est essentiel et aucun n'est décoratif $\mathbf{7}$. Herzog exprime un point de vue opposé à propos des story-boards. Expliquant qu'il ne tourne pas en studio car ceux-ci «offrent rarement des surprises au réalisateur", Herzog développe son propos: "une autre chose que je ne fais jamais - sous peine de détruire inévitablement la spontanéité nécessaire sur un plateau de cinéma - c'est d'établir un story-board de l'action. [...] Les story-boards sont des instruments de lâches qui ne font pas confiance à leur propre imagination et qui sont les esclaves d'une matrice»8. Comme il l'a expliqué ailleurs, sa philosophie du cinéma est basée sur ces éléments imprévisibles et indéfinissables. Décrivant la pratique documentaire de Timothy Treadwell, dont certains enregistrements sont intégrés dans Grizzly Man (Werner Herzog, E.-U., 2005), il mentionne "l'inexplicable magie du cinéma» et soutient que certains moments ne peuvent être saisis qu'à travers l'improvisation. C'est peutêtre pour cette raison que Herzog ne souhaite pas prendre les mesures exactes de ses cadrages. A la différence de maîtres du cadrage contrôlé comme Hitchcock, Stanley Kubrick ou Michael Haneke, Herzog s'intéresse à l'incontrôlable, et son cinéma fait l'éloge du non-planifié. Les 
maîtres du contrôle cinématographique tendent à transformer leurs longs-métrages en des performances de comptabilité fastidieuse: voici l'image et tout ce qui y figure occupe la place qui lui est dévolue. La planification soignée repose sur un processus de différenciation entre le centre et la périphérie; c'est précisément ces notions-là que Herzog désire indifférencier et avec lesquelles il joue.

Que ce soit dans l'intérêt de manifester la présence ou l'absence de contrôle, certains auteurs sont plus enclins que d'autres à diriger l'attention des spectateurs sur les frontières de l'écran et à encourager ainsi à en considérer les limites. Les réalisateurs qui suivent cette tendance le font en inscrivant une image dans le cadre, ou en plaçant un écran bien délimité dans ce que Metz désigne par «le rectangle de vue» 9 . Metz accorde une attention particulière à la façon dont la réalisation de films consiste en une production de cadres, dont Rear Window (Chambre avec vue, Alfred Hitchcock, E.-U., 1954) ou nombre de séquences de films de Rainer Werner Fassbinder, sont caractéristiques. Les fenêtres et les portes divisent le champ visuel en créant un "cadre intérieur" ou un cadre second $\mathbf{1 0}$. De tels gestes témoignent du caractère pleinement artistique de la construction; un écran intérieur ou un cadre second, créant une situation où un "cadre cadre un cadre»11, peut être utilisé pour manifester le talent de cadreur du réalisateur. Cependant, le cadrage peut aussi être utilisé, et cet aspect confère au cinéma de Herzog son caractère d'exception, pour montrer la façon dont tout ce qui apparaît dans le champ ne peut y être contenu. Malgré l'importance des efforts fournis par ceux qui ont travaillé sur ces films, les productions de Herzog excèdent le plus souvent ses projets élaborés, et ses surcadrages attirent l'attention sur l'imperfection et l'instabilité, plutôt que sur la perfection et la stabilité. Une série de plans intentionnellement conçus suivant un story-board représenterait, dans le meilleur des cas pour Herzog, l'espoir déçu d'une production dans laquelle, au nom de l'art, seuls le désordre et l'improvisation prévaudraient. Fitzcarraldo (R.F.A./Pérou, 1982) constitue un des exemples paradigmatiques de cet aspect de son cinéma, la production ayant été mise en difficulté par la défection d'acteurs, les conflits armés aux frontières sud-américaines ainsi que par des accidents ou des maladies. Herzog a délibérément recherché un tel fiasco, et son film est devenu une instance emblématique de ce qui ne peut être contenu. Aurait-il conçu un arrangement préalable de son film plan par plan que cela n'aurait finalement servi qu'à démontrer à quel point rien ne se déroule jamais comme prévu.

Non moins que Fitzcarraldo, Aguirre, der Zorn Gottes (Aguirre, la colère de Dieu, R.F.A., 1972) constitue également un exemple de tournage
9 Voir Christian Metz, L'Enonciation impersonnelle ou le site du film, Paris, Méridiens Klincksieck, 1991, p. 72.

$10 \mathrm{lbid}$.

$11 / d .$, p. 75 . 
12 Herzog confirme qu'il s'agit bien de sa propre main dans la version du film commentée par le réalisateur qui figure sur l'édition américaine du DVD de Aguirre édité par Anchor Bay en 2000. anarchique. Dans une des premières scènes du film, Herzog se livre à une apparition surprenante. Les conquistadors du film, affamés d'or et accompagnés par une quantité innombrable de serviteurs indigènes, tracent leur chemin au travers de sentiers escarpés et boueux, à la recherche de la cité perdue de l'El Dorado. De nombreux éléments de cette séquence d'ouverture signalent le caractère immaîtrisable de la situation : la boue, ni solide ni liquide, forme un terrain sur lequel les protagonistes peinent à évoluer, et il doit avoir été particulièrement difficile de tourner dans ces conditions. Dans ces premières scènes, le paysage semble se répandre par-delà les frontières du cadre. Dans un des plans, alors que l'immense étendue du panorama sylvestre et équatorien se déploie bien au-delà de l'espace délimité par le rectangle de vision, une petite cage à poules artisanale - contenant probablement des volatiles amenés par les conquistadors depuis leur lieu de départ au Portugal - se détache du cortège et tombe dans le précipice, se disloquant au fur et à mesure qu'elle dévale la pente. L'hypothèse que cet évènement ait été mis en scène ou soit au contraire survenu par accident n'a pas pu être vérifiée, tandis que nous assistons à la mort documentée de ces volailles. La cage est cependant une allégorie discrète du rectangle encadré, et renvoie au mouvement incontrôlé d'un cadre dans le cadre, que la caméra a eu la chance de capturer avant qu'elle ne disparaisse.

Plus loin, dans ce qui apparaît comme une tentative de contrôler ces multiples éléments incontrôlables, Herzog pénètre lui-même dans le cadre. Dans cette scène, les serviteurs qui progressent péniblement à travers la boue portent Inez de Atienza, l'amante de Don Pedro de Ursúa, le chef de l'expédition. Celle-ci se déplace dans une chaise à porteurs qui peut être considérée comme un exemple remarquable du concept de surcadrage développé par Metz. Rectangle à l'intérieur d'un rectangle, cadre inclus dans le cadre de vision, les montants de la chaise oscillent et deviennent, de par l'angle qu'ils forment avec l'écran, des substituts à l'image encadrée elle-même, construisant de la sorte une allégorie des aspects les plus instables du projet immaitrisable de Herzog. Juchée sur les épaules des serviteurs enfoncés jusqu'aux genoux dans la boue, la chaise à porteurs se balance fortement, au point qu'elle menace de tomber. A ce moment, on distingue une main, celle de Herzog en l'occurrence, qui pénètre dans le cadre depuis la droite pour s'assurer que la chaise demeure dans le champ de la caméra12. Traversant ainsi les frontières entre le monde réel et celui du film, cette aide garantit que ni le personnage ni Helena Roja (l'actrice qui l'interprète) ne tombe dans la boue. Herzog s'est toujours vanté que, parvenu au stade où ses acteurs sont épuisés ou en situation de danger physique, l'on pouvait 

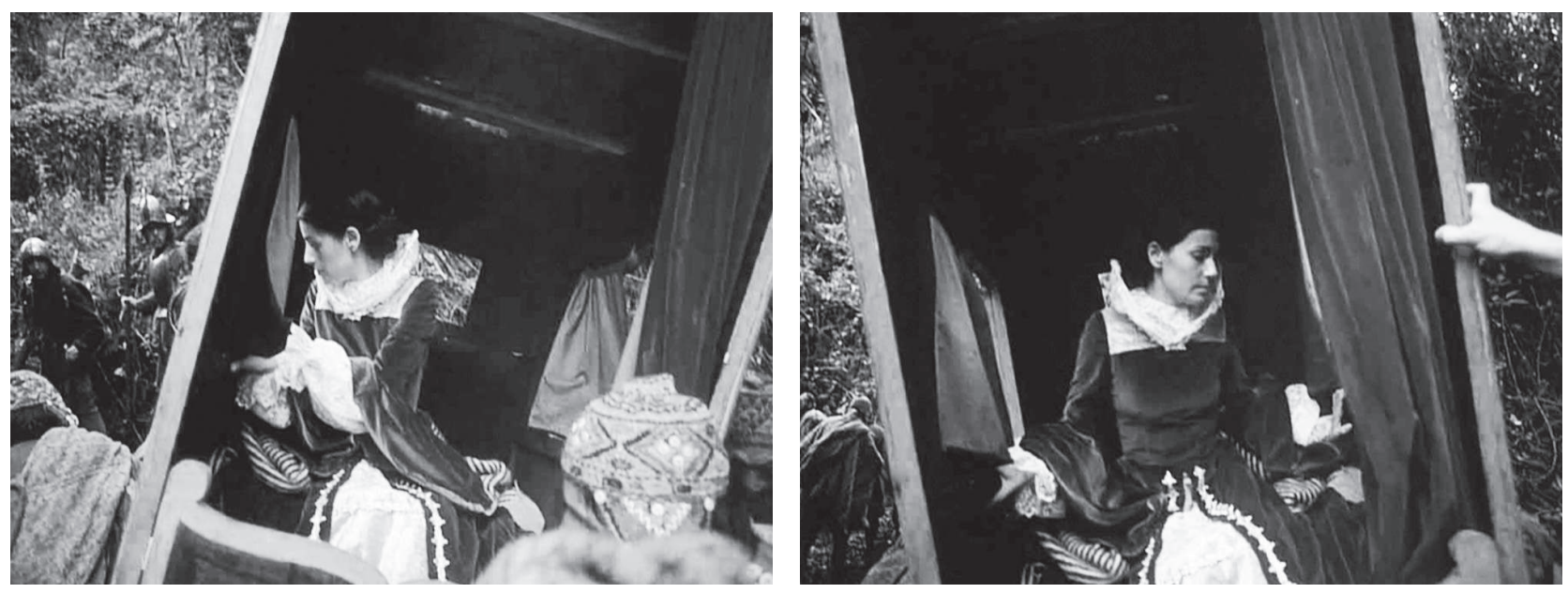

2

lire la tension sur leur visage ; ici, il prend également part à la situation. Plus qu'une signature - plus qu'une empreinte abstraite sur l'image -, la main du réalisateur atteste de sa participation à l'entreprise physique que représente le tournage. Elle a le statut d'un commentaire sur le caractère laborieux du cinéma, mais aussi sur la maîtrise du réalisateur, qui se résume parfois à la question de savoir s'il a les choses «en main". La tentative de ressaisir un objet qui tombe revient à reconnaître sa chute. On peut difficilement imaginer la main de Haneke pénétrant une de ses images pour stabiliser un objet en déséquilibre de la même façon que Herzog s'impose dans le cadre afin de stabiliser cette chaise vacillante (fig. 1 et 2).

Immédiatement après cette intervention, dans le plan suivant, l'objectif de la caméra est visiblement taché par une goutte d'eau sale qui masque en partie le champ de vision. Son maintien est un choix délibéré qui permet d'attirer l'attention sur la présence d'une surface intermédiaire. Quel que soit le degré d'immersion du spectateur - et malgré sa proximité avec l'expérience des conquistadors, potentiellement englué comme eux dans la boue -, cette sensation est inévitablement contrebalancée par la conscience du dispositif filmique. Distincte du statut qu'elle aurait dans un film documentaire dans lequel le réalisateur, présent à l'écran, pourrait nettoyer l'objectif de la caméra, la tache rend visible le support et signale, dans ce film de fiction, qu'il s'agit là d'une surface - et non d'une pure transparence - qui nous sépare de l'action représentée à l'écran $\mathbf{1 3}$. L'apparition de la tache et par conséquent la perturbation du contrat passé avec le spectateur ont été annoncées

13 L'intervention d'un doigt ôtant une tache de l'objectif - en l'occurrence un morceau de nourriture - se produit précisément dans le documentaire de Herzog intitulé Wheel of time (La Roue du temps, All./Autriche/Australie, 2003). Ici, c'est le pouce du caméraman qui se charge du travail. Voir: "Werner Herzog: The Filmmaker as Athlete", entretien avec Jutta Brendemühl, 2003, www.goethe.de/ins/ca/tor/prs/mep/en 2304312.htm, dernière consultation le 10 juin 2013. 
14 Jacques Derrida, La Vérité en peinture, Paris, Flammarion, 1978.

15 Derrida écrit: "Je ne sais pas ce qui est essentiel et accessoire dans une œuvre. [...] Où le cadre a-t-il lieu. A-t-il lieu. Où commence-t-il. Quelle est sa limite interne. Externe. Et sa surface entre les deux limites" (op. cit., p. 73).

16 Voir Derrida, op. cit., pp. 159-160. par la chute de la cage contenant les volailles d'une part, et par le quasieffondrement de la chaise à porteurs d'autre part, rendant nécessaire l'intervention de la main du réalisateur. Chacun de ces moments témoigne de la possibilité d'une perte de maîtrise.

Herzog joue consciemment à ce jeu qui ne repose pas sur la manipulation des lignes de partage entre histoire et discours, ainsi que Hitchcock aurait pu le faire; il s'agit plutôt d'un jeu d'encadrement, interrompant la distinction entre le centre et la périphérie, ou, par extension, la division entre l'œuvre et ses marges. Dans La Vérité en peinture 14, Jacques Derrida explore ces distinctions, notant que les ouvres d'art dessinent généralement une ligne arbitraire entre ce qui est constitutif de l'œuvre et ce qui ne l'est pas. Une différence entre l'œuvre et le cadre qui l'entoure, et plus particulièrement le monde dans lequel elle se situe, est déterminée par ces limites artificielles, qui, nous indiquant où regarder, ont pour but de contrôler le regard du spectateur. De telles divisions, entre ce qui est essentiel (le centre) et ce qui est contingent dans l'œuvre, tendent à servir des motivations spécifiques, historiquement déterminées. L’autorité d'un artiste distingue ce qui relève du centre ou de la périphérie, et ce n'est qu'avec volonté et grâce à un effort considérable que l'on peut maintenir la barrière illusoire entre le monde et l'œuvre ${ }^{15}$. Toutefois, toute ouvre d'art existe toujours au-delà des frontières définies par le cadre, la réalité trouvant son chemin pour y pénétrer, et l'œuvre n'étant jamais complètement séparée du lieu de sa production.

L'analyse de Derrida est spécifiquement centrée sur les mains. Le concept de "préhension" traverse toute son étude, et le verbe fassen [saisir/appréhender] forme une racine essentielle des termes investigués dans la troisième critique kantienne, à laquelle le travail de Derrida fait référence ${ }^{16}$. Des termes comme zusammenfassen [comprendre] et anfassen [appréhender] possèdent une signification particulière dans le contexte des discours sur la perception du sublime dans la nature et dans l'art. Pour Derrida, le corps est la mesure de l'espace inscrit dans un cadre, et les images visuelles sont la mesure de la taille du corps. Ce que la main peut toucher et saisir possède une fonction prothétique et permet à l'œil d'appréhender une distance. Quand Hitchcock arpente la largeur du cadre, il en prend la mesure avec son propre corps. Par contre, Herzog surgit depuis la marge: une main, parmi les nombreux bras ou jambes des indigènes au travail, mesure par là l'étendue du désordre de la scène. L'intervention manifeste, en partie, un désir d'être physiquement intégré dans son film, de participer à l'effort de sa production au lieu de confiner cet effort derrière le récit et les images, comme c'est généralement le cas des films de fiction. Herzog tient à documenter le travail à 
l'œuvre dans la fabrication du film, car, ainsi qu'il le dit fièrement, la réalisation d'un film est une activité physique. Il déclare notamment: "Toute personne qui fait un film doit être, à un certain degré, un athlète, car le cinéma ne trouve pas son origine dans une pensée académique; il vient de vos genoux et de vos cuisses»17. Ce n'est pas une coïncidence si Herzog est célèbre pour troubler les frontières entre documentaires et films de fiction: ses films sont des chroniques de leur propre réalisation, et Herzog, pour cette raison, rend le travail de leur production - et son propre investissement - visible.

Il est possible que ce soit la raison pour laquelle Herzog apparait, tout comme d'autres membres de l'équipe, dans Herz aus Glas et dans Lebenszeichen. Dans ces cas comme dans Aguirre, le travail de la production du film - incluant, dans Herz aus Glas, celui de la manufacture du verre et son transport - est particulièrement mis en évidence. Dans Herz aus Glas, qui se déroule dans le sud de l'Allemagne et en Bohème, le long de ladite "Glasstrasse» ["route du verre»] où la production de verre est à la fois un art et un moyen de subsistance, Herzog dépeint les fondements de sa propre culture. A ce propos, la scène montrant une procession de travailleurs transportant des marchandises sur leur dos et passant devant la caméra a été tournée à Sachrang, la ville dans laquelle Herzog a passé la majeure partie de son enfance.

Dans ce contexte, ces "porteurs de hottes" (aussi appelés Kraxenwaren), comme issus de l'ère proto-industrielle, pourraient être en train d'acheminer le verre couleur rubis qui est spécifique à cette région afin de le vendre de l'autre côté de la frontière $\mathbf{1 8}$. Herzog choisit de s'intégrer à la procession, traversant l'étendue de l'écran, à la façon des autres travailleurs, dans un silence presque complet. En effet, mis à part le son du verre tintinnabulant, la scène ne présente que très peu de sons. La file
17 Herzog on Herzog, op. cit., p. 101 [notre traduction].

18 Pour plus de détails sur cette séquence, voir Noah Heringman, "Herzog's Heart of Glass and the Sublime of Raw Materials", dans Brad Prager (éd.), A Companion to Werner Herzog, Malden, MA: Wiley-Blackwell, 2012, p. 271.

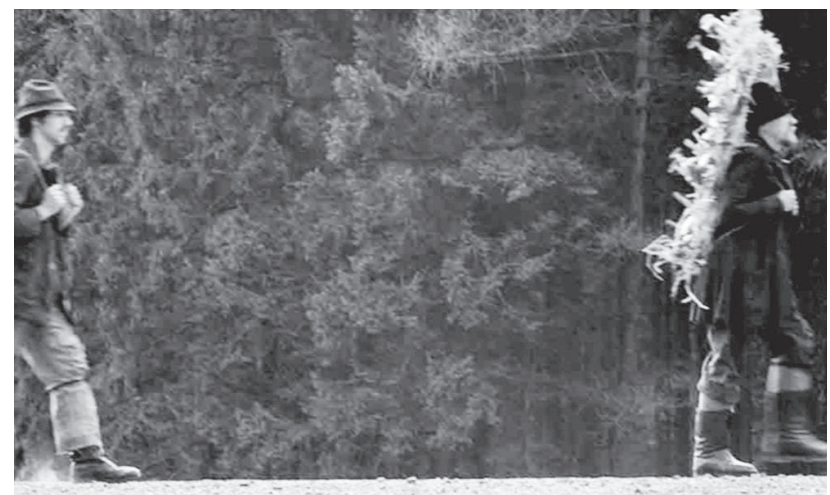

3

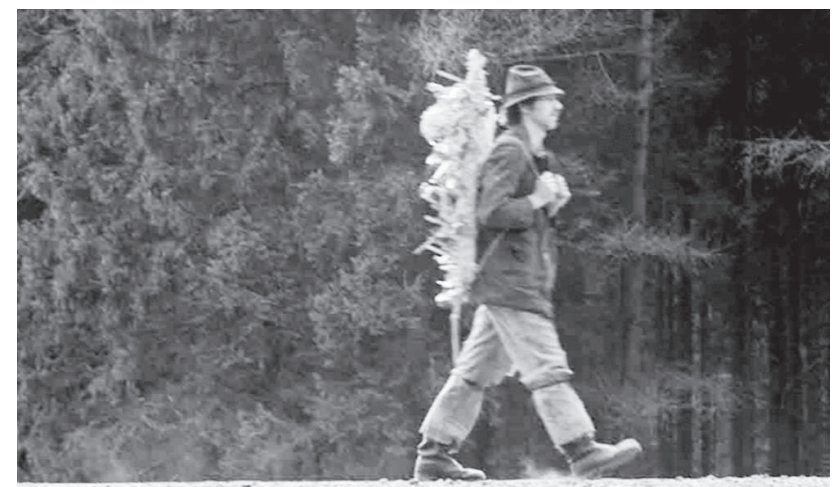

4 
19 Alan Greenberg, Heart of Glass, Munich, Skelling Edition, 1976, p. 155.

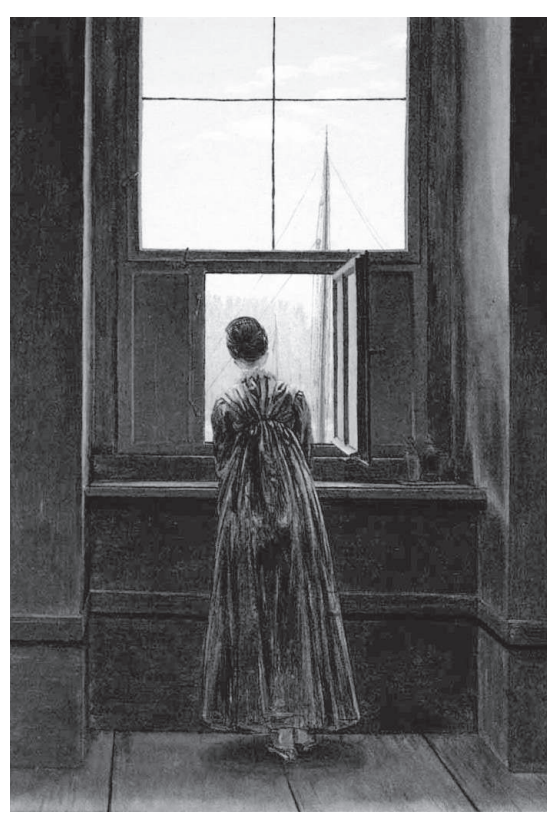

des personnes qui traversent l'écran durant ce plan comprend également Alan Greenberg, un ami du réalisateur qui rédigeait alors la chronique du tournage. Greenberg révèle que d'autres membres de l'équipe étaient présents dans le cortège: Peter van Anft, l'assistant-ingénieur du son, Gunther Freyse, le photographe de plateau, et Joschi Arpa, l'assistant du directeur de production 19. Le fait qu'ils se chargent ainsi de verre montre que les membres de l'équipe sont responsables du travail de la production; le plan devient ainsi emblématique du travail que le film implique (fig. 3 et 4 ).

Contrastant ainsi avec la main intrusive dans Aguirre, la traversée du cadre effectuée par Herzog est plus proche ici de la pratique de Hitchcock, et son implication dans le film - au même titre que la plupart des apparitions de Hitchcock - contribue peu à la diégèse. Bien qu'elle n'apporte quasiment rien à la narration, cette séquence prend davantage de sens si on prend en considération la façon dont elle est encadrée, en particulier le moment qui précède l'apparition de Herzog à l'écran. Les aînés de la ville ont en effet chercher le secret perdu de la fabrication du verre rubis dans un endroit inhabituel: sous les coussins du canapé de la veuve du fabricant de verre. A la recherche du document contenant la formule, ils en viennent à détruire le meuble. Lors du déroulement de ces opérations, la veuve se tient dans l'encadrement de la porte, regardant dehors, et le portrait de cette femme dont on ressent la douleur - elle est sur le point de pleurer - fait écho à l'œuvre du peintre romantique allemand Caspar David Friedrich, en particulier à Frau am Fenster [Femme à la fenêtre], qu'il réalisa en 1822. Dans ce portrait, comme

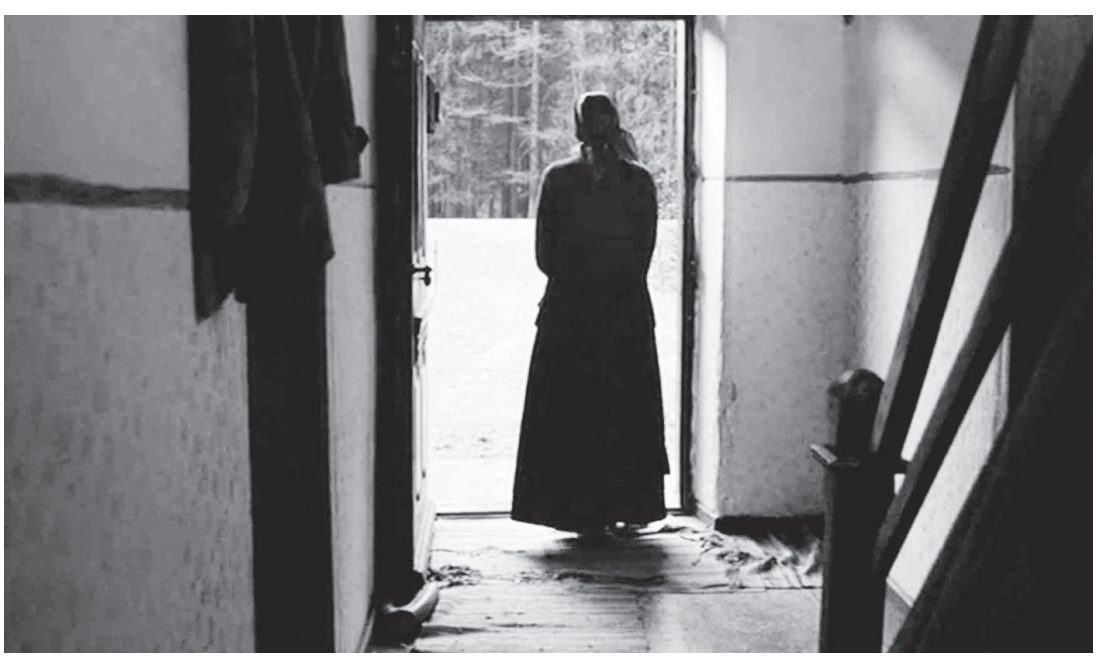


dans la plupart de ses œuvres, Friedrich bloque notre regard, en recourant à un motif typique (dit Rückenfigur), le sujet de la peinture étant vu de dos, en train de contempler la vue. Ici, nous ne pouvons voir ce que la veuve regarde. Les propositions de Friedrich, qui provoquaient en ce sens le spectateur, reposaient sur ce refus: les regards sont subjectifs, et vous ne pouvez pas voir ce que les personnages voient. Mais Herzog, tout en reproduisant la composition de Friedrich, monte cette séquence avec celle des porteurs de verre, dans laquelle il est présent. Grâce au montage, nous voyons ce que la femme voit et Herzog nous entraîne ainsi au-delà du cadre dans le cadre, ici, le cadre de porte qui forme un second cadre dans le rectangle de vision. L'encadrement effectué par le réalisateur inscrit à la fois sa biographie (en particulier ses origines bavaroises) et ses films, par le biais de la référence à Caspar David Friedrich, dans la lignée de l'art allemand 20 (fig. 5 et 6).

Ses «auto-encadrements", propres au style de Herzog, sont présents dès son premier long-métrage, Lebenszeichen. Alors âgé de 24 ans, il apparaît à l'écran avec un air remarquablement juvénile. Au début du film, Herzog sort de l'arrière d'un véhicule militaire, descendant par son ouverture rectangulaire - un cadre dans un cadre -, pour aboutir dans le rectangle de vue. Le personnage principal du film, un soldat allemand du nom de Stroszek, assigné durant la seconde Guerre Mondiale en Grèce, a subi une blessure traumatique grave et fait son entrée en scène sur une civière, littéralement amené dans le cadre du film par Herzog. Une fois de plus, Herzog apparaît aux côtés d'un autre membre de l'équipe du film, en l'occurrence son assistant-caméra Dieter Lohmann. Par ce biais, tous deux sont présentés comme participant aux conditions de possibilité de leur propre film. Au même titre que dans Aguirre, Herzog documente de cette manière sa participation à la réalisation du film, tout en s'impliquant dans la principale thématique du film, puisqu'il porte l'uniforme allemand. Bien que le film traduise par la tangente un rapport avec le passé allemand, la date de sa sortie (1968) coïncidant avec un mouvement global de questionnement de la part des jeunes Allemands sur le rôle de leurs parents pendant la guerre, il constitue surtout un portrait aigu du caractère futile et absurde de la guerre qui mène Stroszek à la folie. En arborant l'uniforme allemand, Herzog signale sa proximité avec les aspects du film qui reposent sur la confrontation avec le passé de l'Allemagne (fig. 7 et 8).

L'apparition de Herzog dans Lebenszeichen effectue précisément ce que nous attendons de lui : il travaille à la production du film, transportant un acteur d'un endroit à l'autre. Il se pourrait que ce soit cet acte de porter une charge - qu'il s'agisse d'Inez de Atienza, du soldat Stroszek
20 Greenberg nous apprend que la femme de Herzog à l'époque avait émis la remarque suivante: "Ce costume fait ressortir le Bavarois en lui [...] Vous regardez le vrai Werner Herzog maintenant" (Greenberg, op. cit., p. 155 [notre traduction]). A propos de la relation spécifique de Herzog avec la Bavière, voir Chris Wahl, "I don't like the Germans': Even Herzog Started in Bavaria", dans Brad Prager (éd.), op. cit., pp. 233-255. 


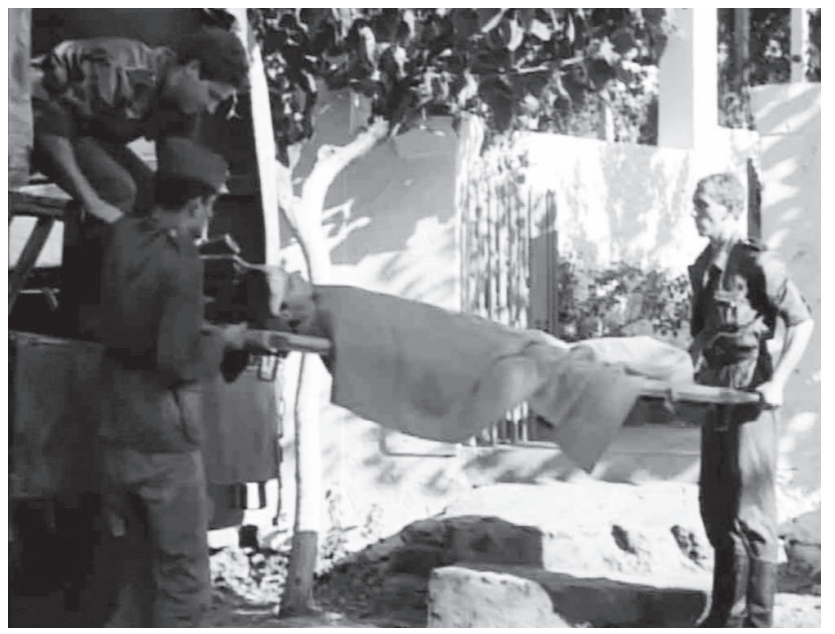

7

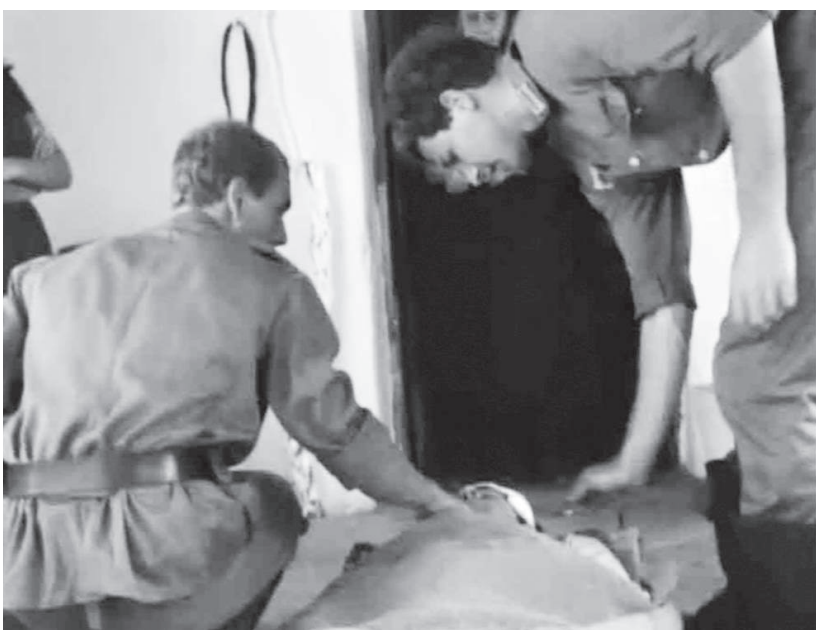

ou du verre rubis - qui définit le mieux sa participation à ses propres films, l'engagement qu'il cherche à saisir à l'écran. Dans Nosferatu: Phantom der Nacht (Nosferatu, fantôme de la nuit, R.F.A./France, 1979), Herzog peut également être aperçu en train de transporter quelque chose, en l'occurrence un nombre impressionnant de rats. En effet, lorsque le film relate le déménagement de son principal protagoniste, Nosferatu, depuis son château en Europe de l'Est jusqu'à la ville de Delft, des rats font également le voyage avec lui. Lorsque le navire et les cercueils du vampire sont arrivés, l'un d'eux est inspecté - avec la terre qu'il renferme - et le pied et la main de Herzog apparaissent à l'écran 21.

21 Herzog imite ici un plan du Nosferatu de F. W. Murnau (All., 1922). L'image - un pied se faisant mordre par un rat dans la terre - est très ressemblante, mis à part l'ajout par Herzog d'un cadre dans le cadre, c'est à dire le cadre du cercueil.
A nouveau, l'entrée dans le cadre s'opère par la marge, et à nouveau, il est ici question de membres, comme c'était le cas dans Aguirre. Ces membres saillent des bords du cadre, révélant sa porosité; cette scène pourrait être interprétée comme un moment typique de surcadrage décrit par Metz: le cercueil, rectangle noir à l'intérieur de l'écran, forme lui-même un cadre, un panneau sombre inséré dans le rectangle de vision. Herzog s'efforce ici de contenir ce qu'il espère réussir à maintenir dans le cadre. Parmi les nombreuses anecdotes relatives à la façon dont Herzog prend des risques pour voir aboutir ses films, celui-ci déclare fièrement que personne d'autre que lui n'avait osé placer son pied au milieu de cette boîte remplie de rats. Il semble d'ailleurs s'y être fait mordre. Cette volonté de se faire mordre, qu'il s'agisse d'une morsure véritable ou d'un moment de jeu, correspond à un geste d'auto-mise en scène : elle confirme à qui voudrait le savoir que Herzog est bien le soldat téméraire du cinéma qu'il prétend être (fig. 9 et 10). 
Les rats incarnent un danger physique pour Herzog mais constituent également une allégorie du caractère incontrôlable du film. A l'instar des volatiles qui chutent de la montagne dans Aguirre, les rats représentent ce qui ne peut être confiné dans une cage ou dans un cadre. L'histoire de leur rôle lors du tournage est célèbre: Herzog s'était fait livrer dix mille rats à Delft pour les besoins du film. Il avait fait peindre les pauvres bêtes en gris, et les avait utilisées comme figurants. Malgré l'accord du Conseil municipal de la ville, leur importation à si grande échelle avait provoqué l'inquiétude de la population de Delft 22. L'histoire de Herzog et des rats fait désormais partie des nombreux récits attestant que la réalité des productions de Herzog tend à déborder les images tournées. Dans une certaine mesure, en amenant ces rats à Delft et en inspirant ainsi la crainte avérée des habitants de la ville, Herzog s'apparentait précisément au vampire qu'il dépeignait. De plus, de façon comparable à la tentative de stabilisation de la chaise à porteurs dans Aguirre, ses membres, émergeant depuis les bords, pour pénétrer dans le cadre, deviennent une indication extra- et intratextuelle de l'effort entrepris par Herzog pour parer une invasion de rats, et endiguer ainsi le risque que le film sorte de ses limites en tentant de maîtriser l'excès inhérent à de telles réalisations. Cette apparition de Herzog, au même titre que toutes les autres, a pour fonction de signaler à quel point la maîtrise est difficile, et comment tous ses projets pourraient échouer; elles montrent que le réalisateur est incapable de contrôler l'ensemble des éléments dont il a pourtant initié la mise en branle.

Les cameos de Herzog dans ses films de fiction illustrent très souvent un effort pour contenir quelque chose dans les limites du cadre, ou pour exercer un contrôle sur ces traces de réalité qui menacent continuellement de surgir. La présence de Hitchcock, par contre, avait une fonction de stabilisation. Elle semble dire: "ce sont les frontières du cadre et
22 L'incident est expliqué en détails dans Herzog on Herzog, op. cit., pp. 156-157. Herzog affirme avoir maîtrisé cette situation pendant toute la durée du film, soutenant que l'équipe de production n'avait pas perdu trace d'un seul des animaux.

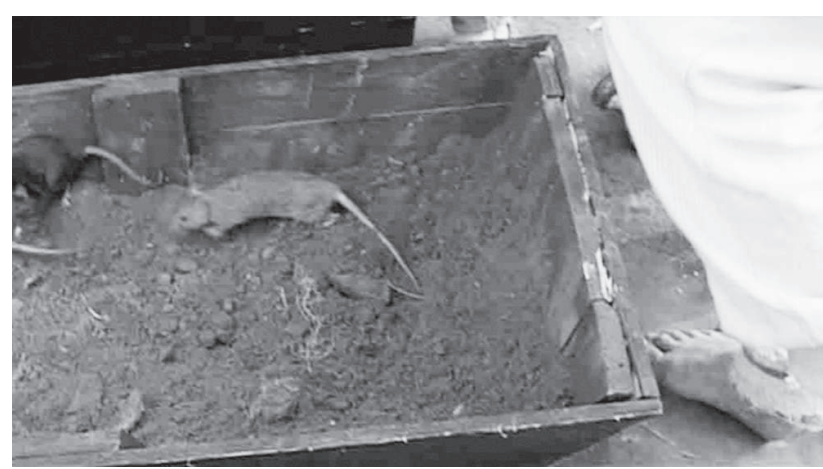

9

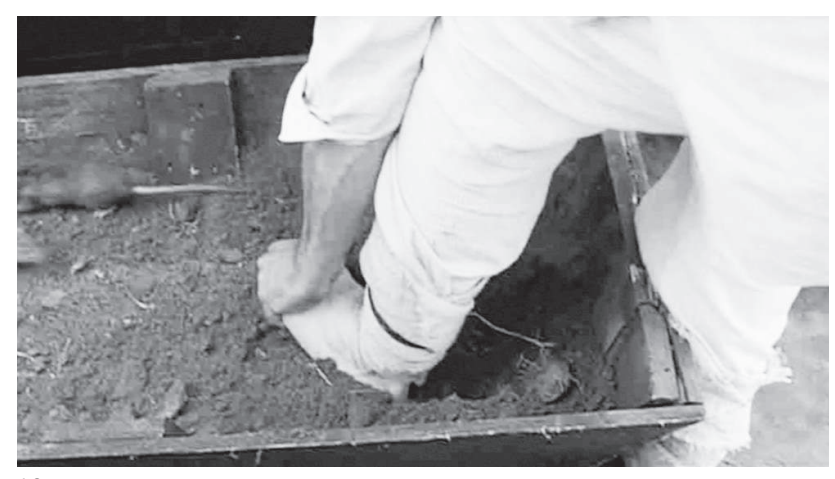

10 
ses justes proportions». Les apparitions délibérées de ce genre affirment que les limites du cadre sont déterminées par le réalisateur. Celles de Herzog sont d'un autre ordre. Elles signifient que le réalisateur n'est qu'une partie d'un processus de production dans lequel tout pourrait potentiellement aller à vau-l'eau, et qui requiert l'intervention nécessaire de nombreuses mains - et pieds - pour y parvenir. Les intrusions de Herzog signalent à la fois la quantité de travail investi et la tentative de maîtriser l'incontrôlable. Ses apparitions renvoient peut-être moins à celles de Hitchcock que de Tiepolo, un de ces célèbres artistes italiens ayant peint sa propre image, dissimulée pour sa part dans les fresques du palais de Würzburg. Cette œuvre, complétée en 1753, ne peut être embrassée d'un seul regard: elle était prévue pour offrir des vues partielles durant la montée du grand escalier. Elle représente quatre continents (l'Amérique, l'Afrique, l'Asie et l'Europe), et les points de vue varient en fonction des différentes positions de l'observateur. Tiepolo s'est représenté lui-même au sein de son chef d'œuvre, mais comme une silhouette parmi d'autres. Il apparaît ainsi relativement insignifiant par rapport à la dimension démesurée du projet dont la vision est, tel qu'il semble l'avoir admis lui-même, trop large pour être appréhendée d'un seul coup d'œil. Les gestes de Herzog sont similaires. Ils reconnaissent la porosité du cadre, son potentiel de rupture et le caractère potentiellement sublime $\mathrm{du}$ film, tout comme l'espoir de l'artiste que son œuvre, peut-être même de par sa propre volonté, pourra s'étendre au-delà de ses frontières présumées et prévisibles.

Traduit de l'américain par Charlotte Bouchez 